\title{
High-resolution tomographic imaging of microvessels
}

\author{
Bert Müller*a,b $^{\text {a, Sabrina Lang }}{ }^{\mathrm{a}}$, Marco Dominietto ${ }^{\mathrm{c}}$, Markus Rudin ${ }^{\mathrm{c}}$, Georg Schulz ${ }^{\mathrm{a}}$, Hans Deyhle , \\ Marco Germann $^{\text {a }}$, Franz Pfeiffer ${ }^{\mathrm{d}, \mathrm{e}}$, Christian David ${ }^{\mathrm{d}}$, Timm Weitkamp ${ }^{\mathrm{f}}$ \\ ${ }^{a}$ Biomaterials Science Center, University of Basel, 4031 Basel, Switzerland; \\ ${ }^{\mathrm{b}}$ Materials Science Institute, Dental School, University of Basel, 4056 Basel, Switzerland; \\ 'Institute for Biomedical Engineering, University and ETH Zürich, 8093 Zürich, Switzerland; \\ ${ }^{\mathrm{d}}$ Paul Scherrer Institute, 5232 Villigen-PSI, Switzerland; \\ ${ }^{\mathrm{e}}$ Ecole Polytechnique Federale de Lausanne, 1015 Lausanne, Switzerland; \\ ${ }^{\mathrm{f}}$ European Synchrotron Radiation Facility (ESRF), 38043 Grenoble, France
}

\begin{abstract}
Cancer belongs to the primary diseases these days. Although different successful treatments including surgery, chemical, pharmacological, and radiation therapies are established, the aggressive proliferation of cancerous cells and the related formation of blood vessels has to be better understood to develop more powerful strategies against the different kinds of cancer. Angiogenesis is one of the crucial steps for the survival and metastasis formation of malignant tumors. Although therapeutic strategies attempting to inhibit these processes are being developed, the biological regulation is still unclear. This study concentrates on the three-dimensional morphology of vessels formed in a mouse tumor xenograft model post mortem. Synchrotron radiation-based micro computed tomography (SR $\mu \mathrm{CT}$ ) could provide the necessary information that is essential for validating the simulations. Using mouse and human brain tissue, the different approaches to extract the vessel tree from $\mathrm{SR} \mu \mathrm{CT}$ data are discussed. These approaches include corrosion casting, the application of contrast agents such as barium sulfate, tissue embedding, all of them regarded as materials science based. Alternatively, phase contrast tomography was used, which gave rise to promising results but still not reaches the spatial resolution to uncover the smallest capillaries.
\end{abstract}

Keywords: post mortem imaging, micro computed tomography, blood vessel staining, vessel formation, tumor growth modeling, x-ray phase contrast, microtomography

\section{INTRODUCTION}

Angiogenesis, which is defined as the formation of blood vessels in vivo, is one of the crucial steps for the initiation, survival, and metastasis formation of malignant tumors. ${ }^{1}$ Although therapeutic strategies attempting to inhibit these processes are being developed, the biological regulation is still blurred. Therefore, computer models ${ }^{2,3}$ have been built up, which should help to recognize the formation of the vascular network and, finally, support the development of more effective strategies against cancer. These models, however, have to be compared with the actually formed blood vessels to prove their validity. In vivo measurements are highly desired but do not provide the necessary spatial resolution to uncover the blood vessels down to the level of the smallest capillaries. Consequently, their value is limited. Currently, the only solution relates to the application of post mortem methods as micro computed tomography $(\mu \mathrm{CT})$ in the conventional absorption contrast mode. It provides the necessary spatial resolution as illustrated in Fig. 1.

In the native state, the tissue consists mainly of water and organic compounds. Thus the contrast between the vessels and the surrounding tissue is extremely weak. The vessels remain invisible. To segment the vessel tree, one has to increase or decrease the X-ray absorption of the vessels with respect to the surrounding tissue. Several approaches are known, which can be categorized into corrosion casting, ${ }^{4-6}$ the vessel staining using highly X-ray absorbing species such as barium sulfate $^{7,8}$ and tissue embedding into appropriate materials such as paraffin. ${ }^{9}$ Besides these materials science-based techniques, phase contrast tomography - a physically based method - leads to sufficient contrast for vessel segmentation. ${ }^{10}$ Here, however, the spatial resolution has not reached the level of the smallest capillaries yet.

This review gives an overview on the application of X-ray tomography to make visible the entire vessel tree of mammals tissues post mortem. It is motivated by the angiogenesis that drives regeneration processes such as wound healing and

Developments in X-Ray Tomography VI, edited by Stuart R. Stock,

Proc. of SPIE Vol. 7078, 70780B, (2008) · 0277-786X/08/\$18 · doi: 10.1117/12.794157

Proc. of SPIE Vol. 7078 70780B-1 
uterine lining, but also the cancer formation. Cancer is still the second most frequent cause of death in our societies, although the therapeutic strategies are constantly refined. Computer models and related simulations of angiogenesis yield a deep insight into the complex phenomena, but require precise validation before final conclusions for alternative cancer therapies can be introduced.

Although the extraction of the entire vessel tree is just one subtask, multi-disciplinary approaches are needed. The life sciences include medicine, cell biology and biochemistry as illustrated in Fig. 1. These disciplines should help to identify the suitable ages and related sizes of tumors that correspond to a volume of up to about one cubic centimeter. The tissue embedding, staining or removal (erosion) can be regarded as materials science approach. The tumor visualization is the task for the engineers or physicists.

Today, clinical CT reaches a spatial resolution no better than a fraction of a millimeter, which is insufficient to reveal the capillaries (see Fig. 2). The available $\mu \mathrm{CT}$ facilities yield generally a three orders of magnitude better spatial resolution and therefore allow visualizing the smallest capillaries. ${ }^{7}$ The experimental setup, however, has to be exhausted in order to quantify the smallest vessels of a cubic centimeter tumor.

Even if the volumetric data allow for the vessel tree segmentation, the data have to be translated to vector-based representations to permit the direct comparison with the related computer simulations. Since the data were obtained post mortem, just one point in time can be compared. The validation becomes, therefore, questionable. In vivo measurements including high-resolution magnetic resonance imaging (MRI) are consequently highly desirable. The registration of the MRI and CT data enables the calibration of the in vivo MRI data by means of the CT data, which usually show two orders of magnitude better spatial resolution. Hence, a significant number of time points can be obtained for the validation or the discard of the computer models. This approach has therefore the potential to generate major impact on the developments of clinical tumor treatments.

The injection of human cancer cells in nude mice permits the indirect examination of the human tumor growth. Because in nude mice the thymus gland is removed, the immune system is inhibited and the tumor growth advanced. Since the smallest blood vessels in mice are much smaller than in humans (cp. Fig. 2), the visualization of the vascular network is a more challenging task.
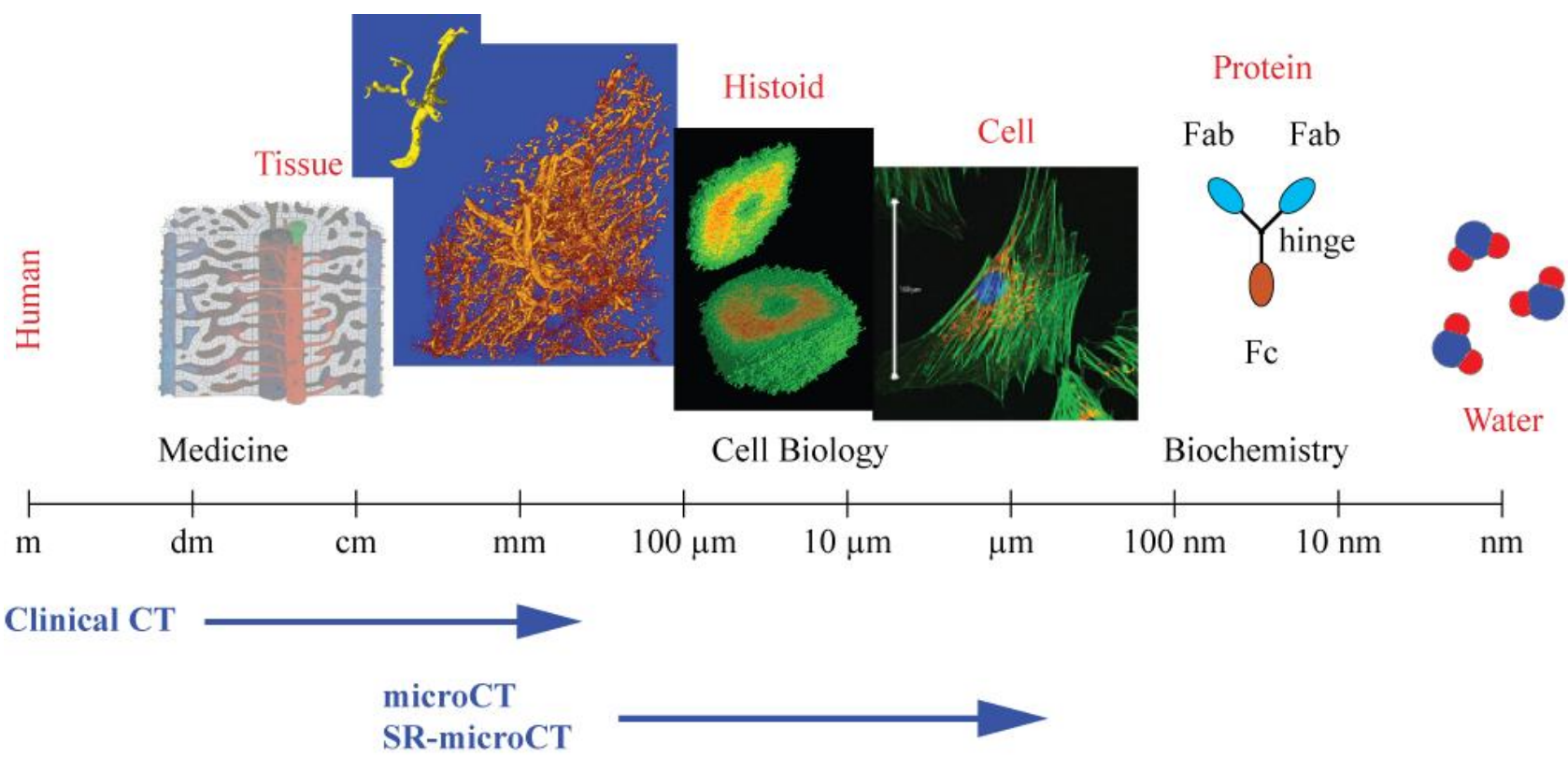

Fig. 1. Life sciences include medicine, cell biology, and biochemistry. They cover the length scale from meter to nanometer. Clinical CT currently reaches the sub-millimeter range. Micro computed tomography usually can resolve micrometer features. For small specimens and dedicated experimental set-ups, several teams have presented sub-micrometer resolution. 


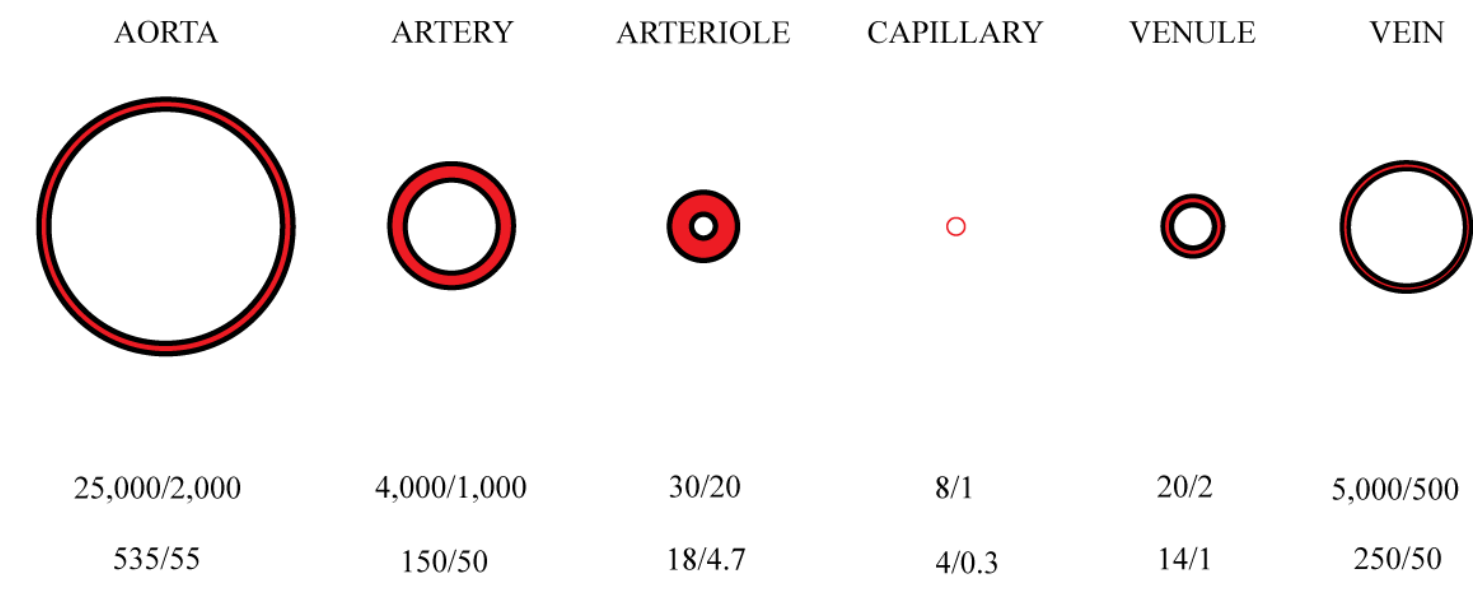

\begin{tabular}{|c|c|c|c|c|c|c|}
\hline Human & $25,000 / 2,000$ & $4,000 / 1,000$ & $30 / 20$ & $8 / 1$ & $20 / 2$ & $5,000 / 500$ \\
\hline Mouse & $535 / 55$ & $150 / 50$ & $18 / 4.7$ & $4 / 0.3$ & $14 / 1$ & $250 / 50$ \\
\hline
\end{tabular}

Fig. 2. The typical diameters/wall thicknesses of blood vessel for humans and mice are given in micrometers. The smallest capillaries have diameters of a very few micrometers and a wall thickness of about one micrometer.

\section{MATERIALS AND METHODS}

\subsection{Specimen preparation}

Tumor cells of colon cancer, cell line C51, were injected into female nude mice (Balb/c) with a weight of about $25 \mathrm{~g}$ in strict adherence to the Swiss law for animal protection. The mice were sent to death by $350 \mathrm{ml}$ ketamin. Subsequently, the brain and the tumor were isolated, fixed in 4\% para-formaldehyde and transferred into Eppendorf containers for the imaging using $\mathrm{SR} \mu \mathrm{CT}$.

The human brain was dissected from a donator about one day after natural death. After fixation in formalin, it was cut into rectangular blocs and put into a container for SR $\mu$ CT-measurements. The paraffin embedding of human brain tissue was described in detail. ${ }^{9}$

\subsection{Staining of vessels}

The contrast agent was a suspension of barium sulfate and physiological solution with a concentration of $80 \mathrm{~g} / \mathrm{l}$. The suspension was filtered (pore size $40 \mu \mathrm{m}$, BD Falcon, USA) to obtain particles with dimensions comparable to the diameters of micro-vessels. Before injection, the suspension was made homogeneous using the ultrasonic bath Sonorex Digital 10P, Bandelin at the temperature of $37{ }^{\circ} \mathrm{C}$ for a period of $10 \mathrm{~min}$. The mice were anesthetized by an intraperitoneal injection of $350 \mu \mathrm{m}$ Ketamine/Xylazine. Subsequently, the animal was perfused with $250 \mathrm{ml}$ heparin to avoid thrombosis. Finally, $10 \mathrm{ml}$ barium sulfate suspension was injected via the left ventricle of the heart applying the peristaltic pump (Watson Marlow $101 \mathrm{U} / \mathrm{R}$ ) as described by Myojin et al. ${ }^{11}$

\subsection{Synchrotron radiation-based micro computed tomography}

The SR $\mu \mathrm{CT}$-measurements were performed at the beamline BW 2 (HASYLAB at DESY, Hamburg, Germany), at the beamline TOMCAT (SLS at PSI, Villigen, Switzerland) and at the beamline ID 19 (ESRF, Grenoble, France).

The experiments at the beamline BW 2 took advantage of the standard setup for absorption contrast tomography ${ }^{12}$ using the photon energy of $10 \mathrm{keV}$. The setup is operated by the GKSS research center. For the present study, 3 datasets at different sample heights each with 721 projections were acquired by rotating the sample in steps of $0.25^{\circ}$ from 0 to $180^{\circ}$. The reconstruction was performed slice-by-slice with the filtered back-projection algorithm. In order to improve the density resolution, the data, recorded with a pixel size of $2.1 \mu \mathrm{m}$, were binned by a factor of two before reconstruction. ${ }^{13}$ The spatial resolution was determined by means of a gold edge as described previously. ${ }^{14}$ It corresponded to $4.25 \mu \mathrm{m}$.

The SR $\mu \mathrm{CT}$-measurements at TOMCAT were carried out in local absorption contrast mode using the photon energy of $18 \mathrm{keV}$ (band width $2 \%$ to 3\%) and the exposure time of $350 \mathrm{~ms}$ per projection. The samples were rotated in steps of $0.12^{\circ}$ between $0^{\circ}$ and $180^{\circ}$ to record the projections, which serve for the reconstruction by the filtered back-projection 
algorithm. The shutter was closed during CCD readout to avoid bubble formation in the liquid around the sample. A pixel size of $3.7 \mu \mathrm{m}$ had been chosen, which does principally allow visualizing the smallest vessels in mice.

Phase contrast tomography data were acquired at the beamline ID 19. The data acquisition was based on the grating interferometer ${ }^{15}$ at the photon energy of $26.0 \mathrm{keV}$ during $1 \mathrm{~s}$ per projection. Each projection consists of 8 images over 2 grating periods. The specimen was rotated in steps of $0.36^{\circ}$ between $0^{\circ}$ and $180^{\circ}$. The Talbot distance between the gratings with periods of $2 \mu \mathrm{m}$ (analyzer) and almost $4 \mu \mathrm{m}$ (beam splitter with $3.99 \mu \mathrm{m}$ period) was set to $376 \mathrm{~mm}$. Using a binning factor of two, the voxel size corresponded to $15 \mu \mathrm{m}$. The other SR $\mu$ CT measurement presented in Fig. 9 was obtained with the same experimental set-up, but shorter exposure time $(0.5 \mathrm{~s}), 4$ images per grating period and sample rotation steps of $1.2^{\circ}$ between $0^{\circ}$ and $360^{\circ}$.

\subsection{Data visualization}

For the 3D visualization of the blood vessels the software VG Studio Max 1.2 (Volume Graphics, Heidelberg, Germany) was applied. Thresholds and colors were manually selected to elucidate the features of interest.

\section{RESULTS AND DISCUSSION}

Native brain or tumor tissues do not provide the necessary X-ray attenuation contrast differences between vessels and surrounding tissue to extract the entire vessel tree. Therefore, the tissues have to be prepared in the appropriate manner. The preparation procedures can be divided into corrosion casting, vessel staining with highly X-ray absorbing species and tissue embedding. Alternatively, phase contrast imaging can be used, which usually provides much better contrast for soft tissues than absorption contrast, but is still restricted in spatial resolution.

\subsection{Paraffin embedding for vessel extraction}

To increase the contrast between the vessels and the surrounding brain tissue the sample was embedded into paraffin. Standardized paraffin embedding is well established and relatively easy to perform. It is assumed that paraffin penetrates the brain tissue without full penetration of the vessel walls resulting in (partially) empty vessels. The reconstructed slice (Fig. 3, left image) indicates that a stronger absorbing layer, associated with the blood vessel walls, surrounds the cavities. This behavior simplifies the intensity-based segmentation of the vessel tree, which is shown in the 3D representation of the segmented cavities reproduced on the right of Fig. 3. It should be noted that even the smallest blood vessels, i.e. the capillaries are uncovered, because the voxel size corresponds to about $2 \mu \mathrm{m}$ and the spatial resolution of $4 \mu \mathrm{m}$ is equal or smaller than the diameters of the capillaries in human brain.
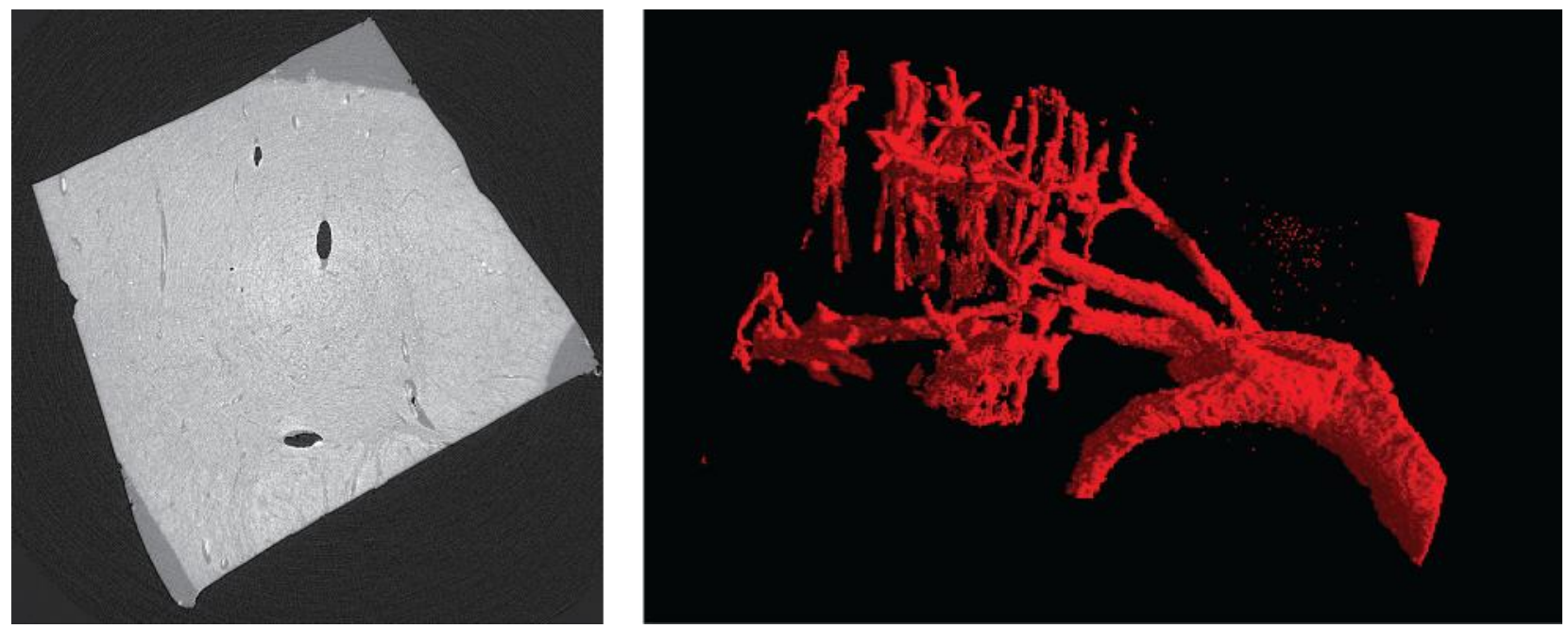

Fig. 3. Since the paraffin does not fully penetrate through the vessel walls, the vessels exhibit less X-ray absorption and can be intensity-based segmented using the suitable threshold. 
This method, however, has different drawbacks. First, individual steps of the embedding procedure are temperature sensitive. Second, the adaptation of the procedure to other kinds than human brain tissue is unknown, which might lead to extensive optimization protocols. Third, the tissue shrinkage is not measured yet and can, therefore, only be estimated.

\subsection{Corrosion casts}

The procedures to generate corrosion casts are well established in the scientific literature. ${ }^{5,6}$ This approach supported our knowledge on the variety of blood vessel structures present in the different parts of the human body. ${ }^{16}$ Nevertheless, this kind of vessel tree preparation has many disadvantages difficult to be mastered. First, the corrosion casting is time consuming and relies on dangerous chemicals. Second, one has to select the suitable viscosity of the appropriate resin to fill the smallest vessels. This selection has concentrated on methyl methacrylate resins that give rise to very fragile constructs, which are usually damaged before the imaging by secondary electron microscopy or CT is performed. Alternatively, elastic constructs made out of polyurethane ${ }^{5}$ have been built, but their flexibility is probably problematic for tomographic imaging (rotating sample) and differs from the one of native tissue. Third, although some studies have been carried out on the shrinking behavior, ${ }^{5}$ the results are restricted to the global structures and estimates on the vessel diameters for example are rather questionable.

The imaging of corrosion casts provides many anatomical features of the vessel tree. The vessel tree of an entire rat brain and even the organization of tiny capillaries of sub-volumes became visible. ${ }^{5}$ The approach was previously applied for the validation of neo-vascularization phenomena of the porcine myocardium, ${ }^{6}$ which plays a crucial role for the treatment of cardio-vascular diseases. Here, the technique supports the validation of results of conventional histology. ${ }^{6}$

\subsection{Vessel staining by highly $\mathrm{X}$-ray absorbing species}

The blood vessels can be filled by species that cannot penetrate though the vessel walls. Salts almost insoluble in aqueous environment such as calcium, strontium or barium sulfate are perfect materials. ${ }^{7}$ Their particle size has to be smaller than the capillaries diameters. Carrier media could be water or resin. The JB-4 (Polysciences, Inc., Warrington, USA) belongs to the well-suited carrier media and also allows monitoring the filling since the color of the tissue changes to yellow-white (cp. Fig. 4). The photographs in Fig. 4 show the preparation procedure of porcine myocardium, which includes the filling through a major vessel, the cutting of tissue for the SR $\mu \mathrm{CT}$ measurements and the final embedding into the JB-4 resin.

For mice such a procedure is almost impossible since even the major vessels are too small. The only exception is the main vein of the tail. Filling though this vein, however, almost exclusively enriches the particles in the mouse lung. ${ }^{17}$ Hence, one has to go via the left ventricle of the heart applying a peristaltic pump. The images in Figs. 5 and 6 show the success of the barium sulfate filling of the brain and tumor vessels in mice.
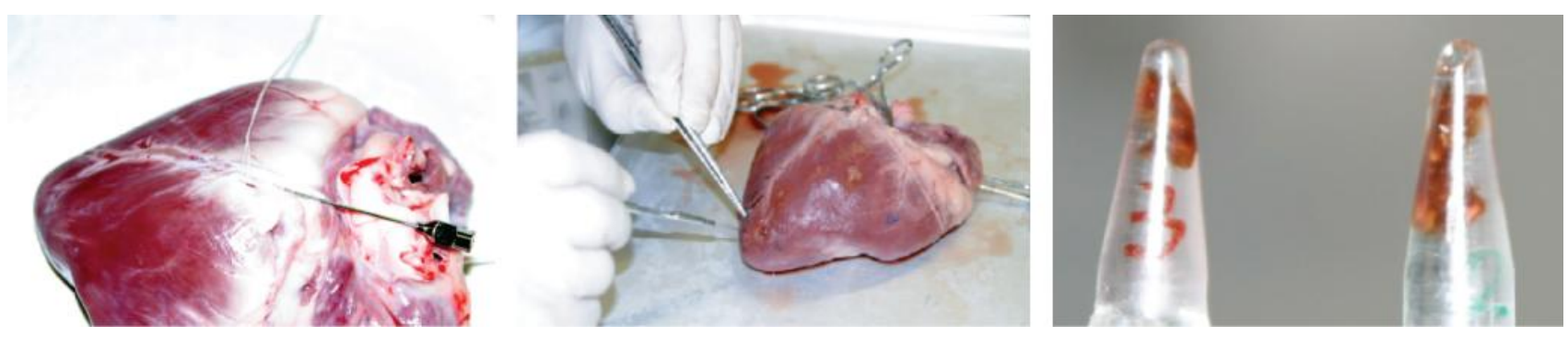

Fig. 4. The vessels of the porcine myocardium can be stained with sulfate particles in the JB-4 monomer solution. The color of the tissue changes to white-yellow, which easily allows selecting the tissue to be embedded for the SR $\mu \mathrm{CT}$ measurements. (The photographs were taken by J. Fischer.) 

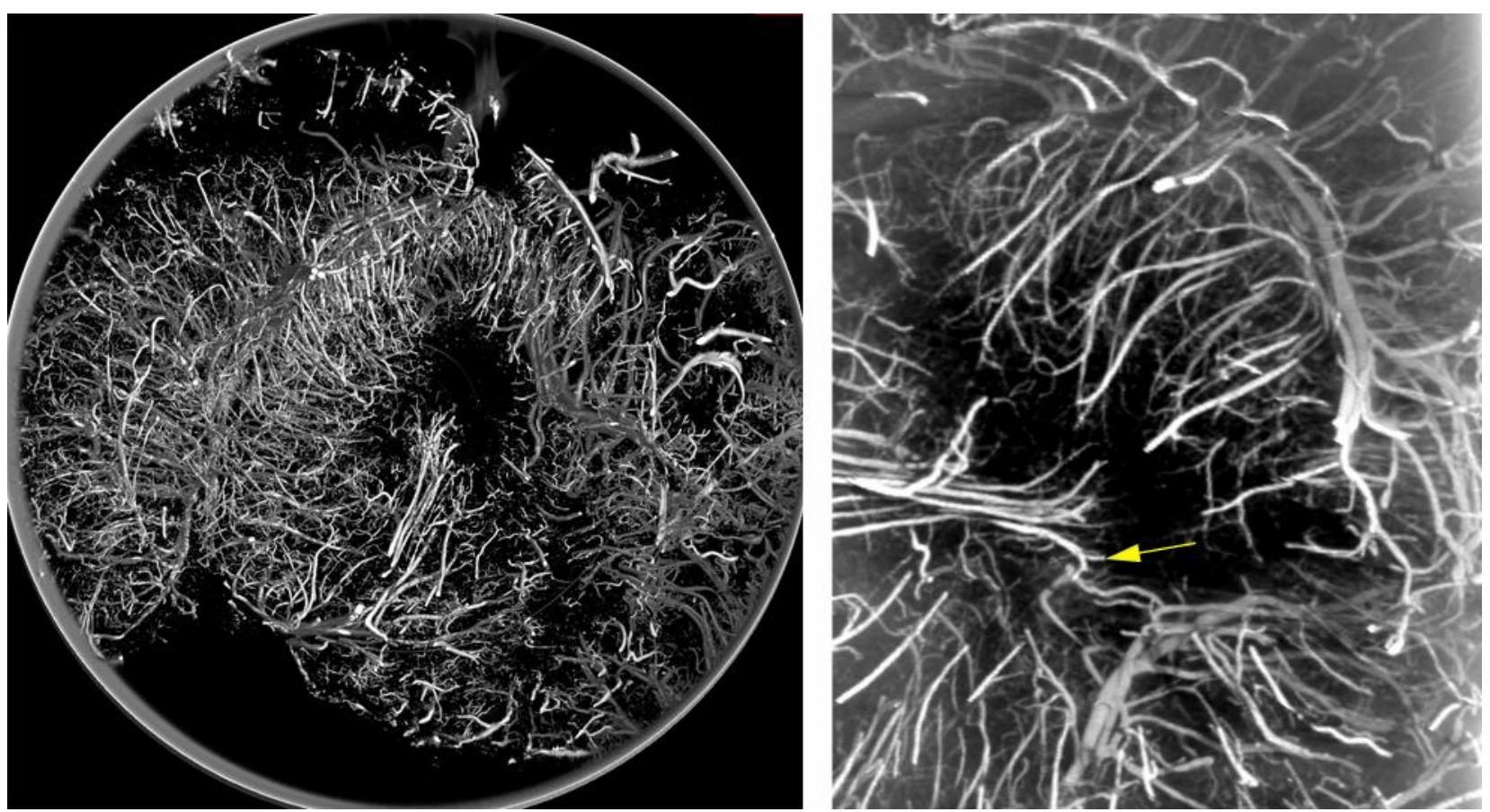

Fig. 5. The vessels of the mouse brain are well reproduced after barium sulfate staining using the tomography set-up of TOMCAT. The diameter of the local tomography data corresponds to $7.5 \mathrm{~mm}$ (left image). The arrowhead in the right image is directed to a $40 \mu \mathrm{m}$-wide blood vessel.

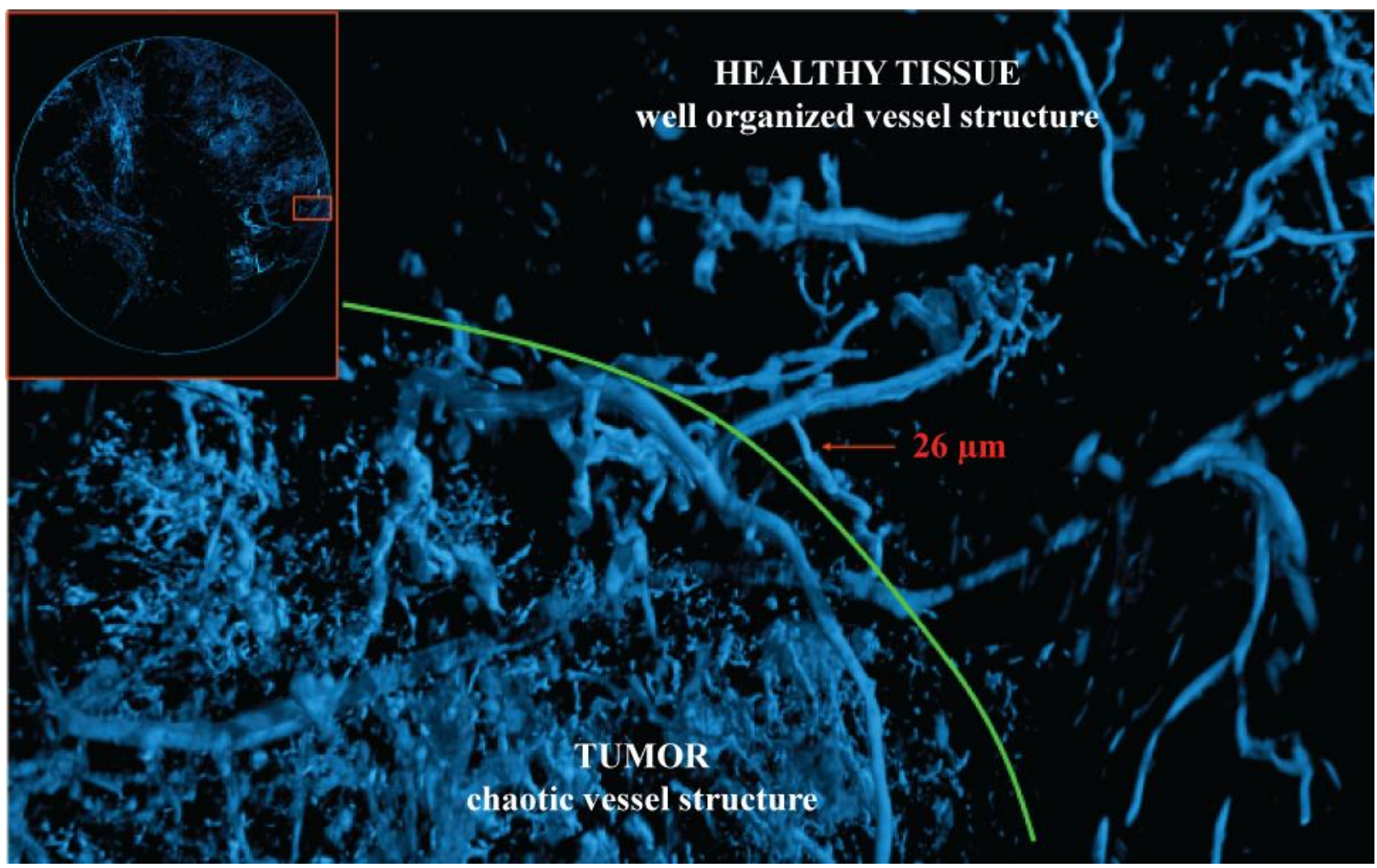

Fig. 6. The 3D representation shows the different morphology of vessels in the tumor and healthy tissues. The density of the tumor vessels is much higher. Many vessels seem to be interrupted most probably as the result of the barium sulfate particle sedimentation. 
Unfortunately, the carrier media and the salts have very different physical densities resulting in sedimentation of the salt particles. This sedimentation of the suspension impedes the vessel visualization by $\mu \mathrm{CT}$ and produces 'disconnected' vessels. Furthermore, the appropriate density of micro- or nano-particles has to be transferred to the tissue of interest. Therefore, it is rather simple to fill the lung or the brain but much more demanding to reach the tumor.

In the final states of tumor growth, necrotic parts occur. The necrotic stage contains vessels, which are not integrated into the blood flow anymore. They could be somehow open. Therefore, one observes dark-red regions associated with coagulated blood in the necrotic part. Such a situation is made visible in Fig. 7. The barium sulfate stain left the vessels at certain points, which are highlighted by red circles.
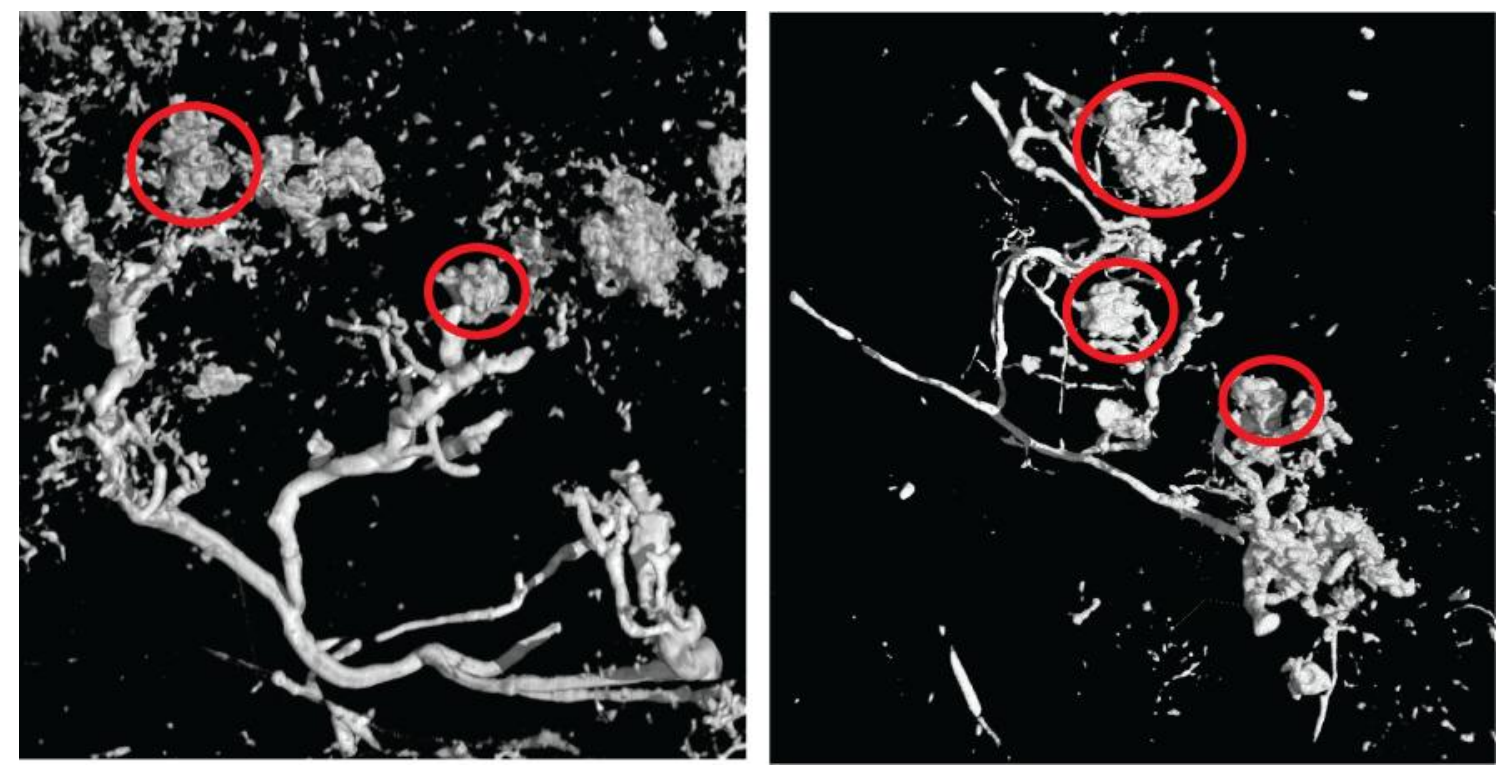

Fig. 7. The vessels within the tumor occasionally exhibit ends with a conglomerate of staining material. This behavior is characteristic for the tumor tissue and related to the dark red color of tumors in the final state. At this stage, the vessels seem to be open and not integrated into the blood flow anymore.

\subsection{Visualization of unstained blood vessels using grating interferometry}

Phase-contrast often shows higher sensitivity than absorption contrast X-ray computed tomography. ${ }^{18}$ It is especially suitable to detect differences in soft tissues. Consequently, it was also successfully applied to uncover the blood vessels for example in brain tissue of a rat. ${ }^{10}$ Figure 8 shows the vessels in a part of the mouse brain that can be easily segmented from the raw data. More sophisticated segmentation tools have to be used to make transparent the brain-water interface that exactly shows the phase shift of the vessels within the brain. 

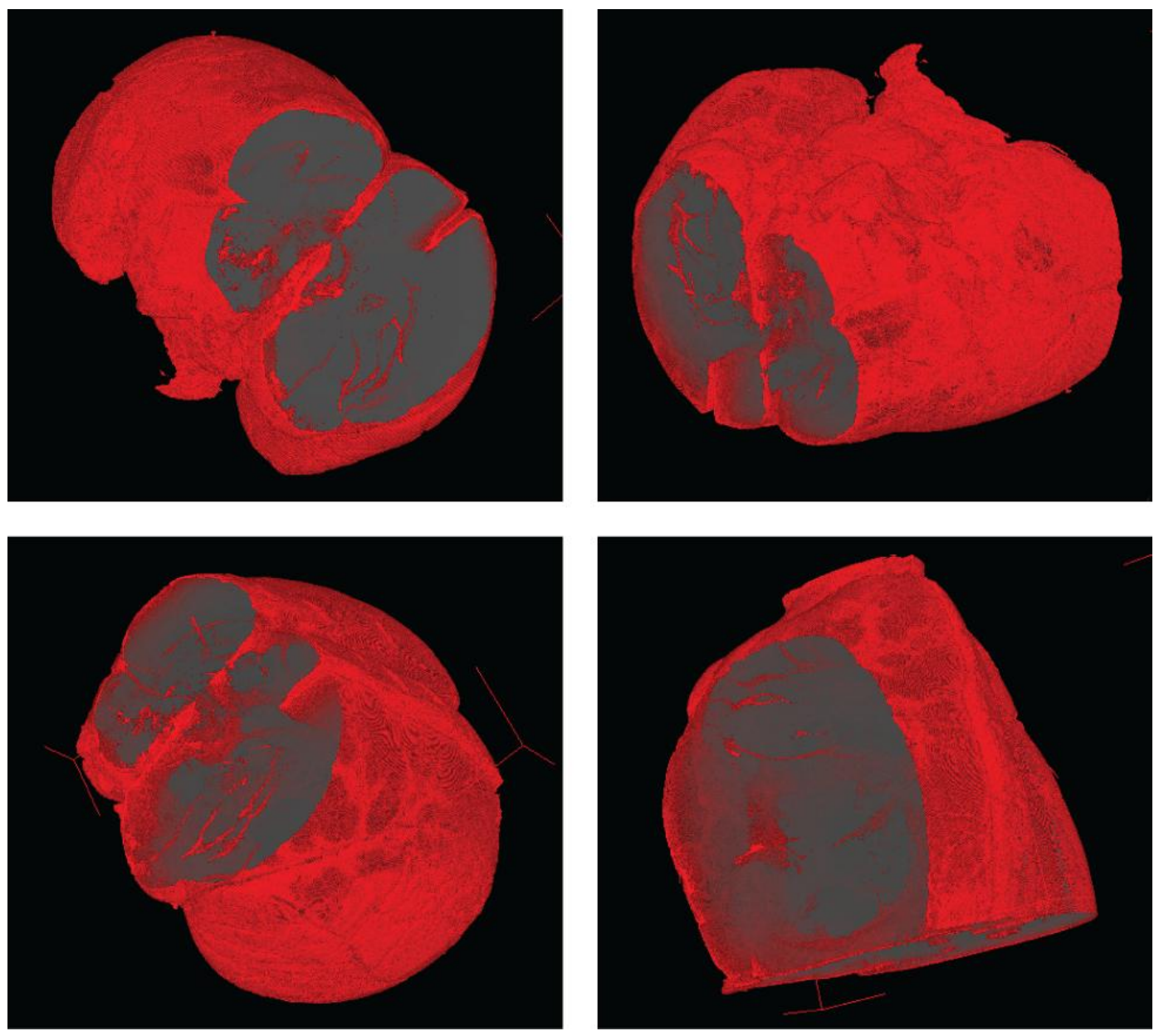

Fig. 8. The 3D images of the unstained mouse brain in phosphate buffer solution were generated by means of grating interferometry and show distinct blood vessels. The intensity-based segmentation of the blood vessels is complicated, since the interface brain tissue-liquid exhibits the same phase shift.

In Fig. 9, the vessels of a part of human brain are uncovered. One can even differentiate between the blue-colored and red-colored vessels that show significantly different phase shifts. Blue-colored regions exhibit less phase shift than the brain tissue itself, whereas the red-colored regions show larger phase-shift than the brain tissue and correspond to the phase-shifts of the surrounding water. More detailed segmentation procedures have to be applied in order to remove the red-colored surrounding water. Vessels with diameters as small as one voxel can be identified. It is, however, still unclear, whether this technique reaches the spatial resolution to uncover the smallest capillaries or not.

With the knowledge that the human brain contains the vessel-like structures with a significantly smaller phase shift than the brain tissue, we have tried to detect similar microstructures in the mouse brain. Indeed, one recognizes some vessels but many of them seem to be so thin that they are not well detected with the pixel size chosen. This means higher spatial resolution is necessary to identify the entire vessel tree of the mouse brain. 


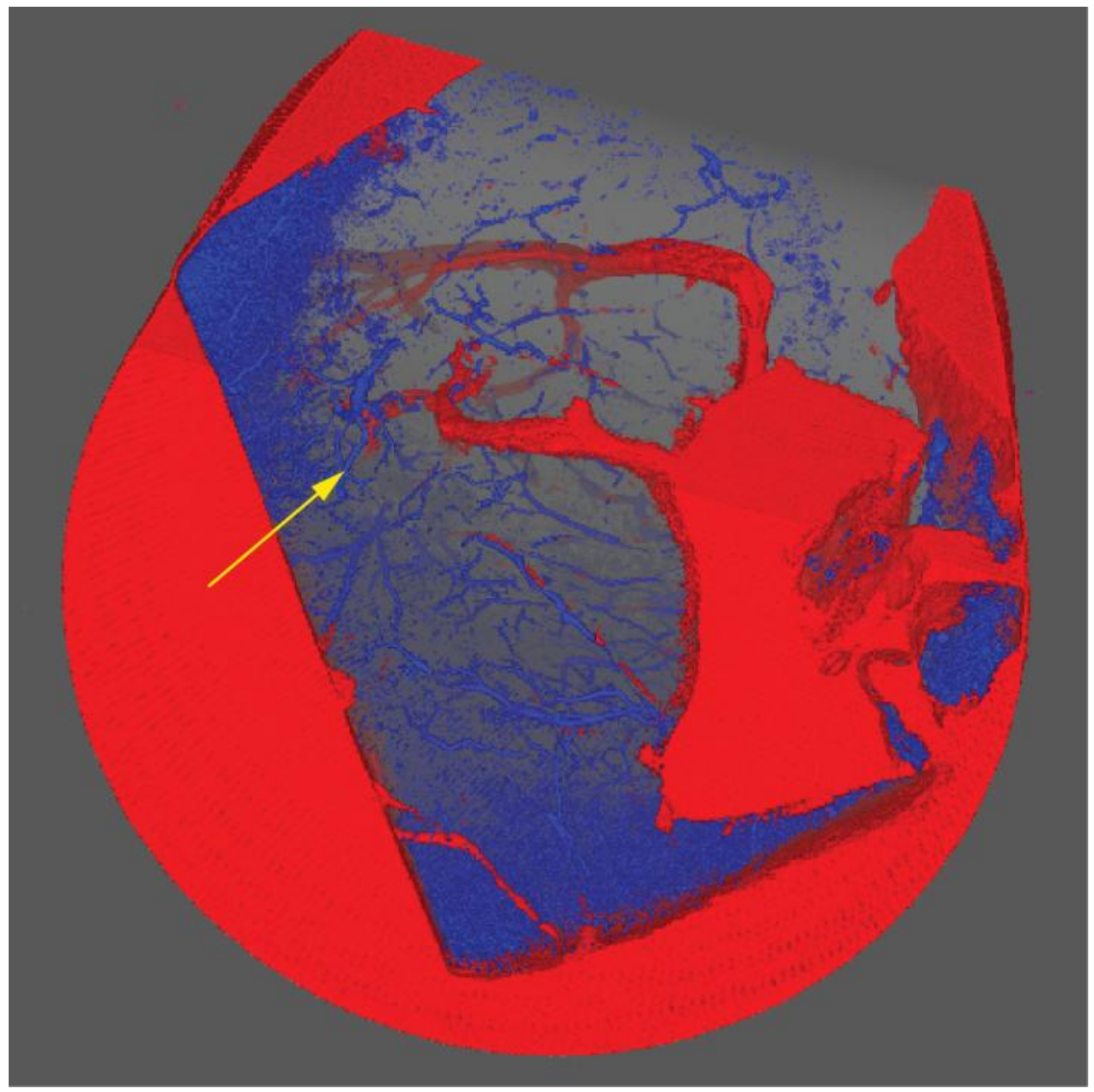

Fig. 9. The 3D representation of a part of the human brain obtained from phase-contrast tomography shows brain vessel structures of different phase shifts, which are represented in red and blue. The arrowhead indicates a vessel of $75 \mu \mathrm{m}$ in diameter.

\section{CONCLUSIONS}

The vascular system has fascinated the scientists for centuries. Micro computed tomography as a nondestructive 3D technique enables us to make visible the vascular system down to the capillary level. The application of the absorption contrast mode (attenuation contrast) relies on materials that are necessary to produce a difference in absorption between tissue and vessels. Although these approaches are continuously improved, they are often difficult to be established and the reproducibility is almost impossible for the smallest capillaries. Therefore, one can state the materials-based methods yield still imperfect results regarding the entire vascular system.

Phase contrast imaging has been known for decades and has been available to users since the end of 1990s. More recently the grating interferometer has been applied for X-ray tomography and already provided surprisingly valuable results for soft tissues. The phase-contrast techniques are relatively time consuming, especially if high spatial resolution is required. The tomography based on the grating interferometer has provided promising results with respect to contrast and spatial resolution and only a small step forward has to be made in order to reach the goal of the quantification of the vascular tree down to the smallest capillaries.

Even if the visualization of the entire vessel tree will be fully solved, powerful computer code has to be developed to quantify the parameters necessary to validate computer models on angiogenesis, which finally will support searching for alternative cancer treatments.

The high-resolution in vivo MRI provides the flow through the vessel system. The diameters of the smaller vessels, however, are not accessible. Consequently, the post mortem SR $\mu$ CT-measurements can be applied to calibrate the MRI data after appropriate registration. The combination of the different modalities with advanced software tools gives rise to 
a comprehensive view of angiogenesis including potential routes to successfully treat different kinds of cancer and to improve the sophisticating patient treatments.

\section{ACKNOWLEDGEMENTS}

The financial support of the Swiss National Science Foundation via the NCCR Co-Me is gratefully acknowledged. The authors thank the beamline scientists F. Beckmann in Hamburg and M. Stampanoni in Villigen for their valuable support. The experiments at the beamlines were performed within approved proposals: II-20060035 EC at HASYLAB/DESY, 20080411 at SLS, and MD-328 at ESRF.

\section{REFERENCES}

[1] Hanahan, D. and Weinberg, R.A., "The hallmarks of cancer", Cell 100, 57-70 (2000).

[2] Lloyd, B.A., Szczerba, D. and Szekely, G., "A Coupled Finite Element Model of Tumor Growth and Vascularization", LNCS 4792, 874 (2007).

[3] Szczerba, D. and Szekely, G., "Computational model of flow tissue interactions in antisusceptive angiogenesis", J. Theo. Biol. 234 (1), 87-97 (2005).

[4] Heinzer, S., Krucker, T., Stampanoni, M., Abela, R., Meyer, E.P., Schuler, A., Schneider, P. and Müller, R., "Hierarchical microimaging for multiscale analysis of large vascular networks", NeuroImage 32, 626-636 (2006).

[5] Krucker, T., Lang, A. and Meyer, E.P., "New polyurethane-based material for vascular corrosion casting with improved physical and imaging characteristics", Microsc. Res. Tech. 69, 136-147 (2006).

[6] Kwon, H.M., Hong, B.K., Jang, G.J., Kim, D.S., Choi, E.Y., Kim, I.J., McKenna, C.J., Ritman, E.L. and Schwartz, R.S., "Percutaneous transmyocardial revascularization induces angiogensis: A histologic and 3-dimensional micro computed tomography study ", J. Korean Med. Sci. 14, 502-510 (1999).

[7] Müller, B., Fischer, J., Dietz, U., Thurner, P.J. and Beckmann, F., "Blood vessel staining in the myocardium for 3D visualization down to the smallest capillaries", Nucl. Instrum. Meth. B 246, 254-261 (2006).

[8] Plouraboue, F., Cloetens, P., Fonta, C., Steyer, A., Lauwers, F. and Marc-Vergnes, J.-P., "X-ray high-resolution vascular network imaging", J. Microsc. 215 (2), 139-148 (2004).

[9] Müller, B., Germann, M., Jeanmonod, D. and Morel, A., "Three-dimensional assessment of brain tissue morphology," Proc. SPIE. 6318, 63180301-9 (2006).

[10] Pfeiffer, F., Bunk, O., David, C., Bech, M., Le Duc, G., Bravin, A. and Cloetens, P., "High-resolution brain tumor visualization using three-dimensional x-ray phase contrast tomography", Phys. Med. Biol. 52, 6923-6930 (2007).

[11] Myojin, K., Taguchi, A., Umetani, K., Fukushima, K., Nishiura, N., Matsuama, T., Kimura, H., Stern, D.M., Imai, Y. and Mori, H., "Visualization of intracerebral arteries by synchrotron radiation microangiography", Am. J. Neuroradiol. 28, 953-957 (2007).

[12] Beckmann, F., "Microtomography using synchrotron radiation as a user experiment at beamlines BW2 and BW5 of HASYLAB at DESY", Proc. SPIE 4503, 34-41 (2002).

[13] Thurner, P., Beckmann, F. and Müller, B., "An optimization procedure for spatial and density resolution in hard Xray micro-computed tomography", Nucl. Instrum. Meth. 225 (4), 599-603 (2004).

[14] Müller, B., Thurner, P., Beckmann, F., Weitkamp, T., Rau, C., Bernhardt, R., Karamuk, E., Eckert, L., Brandt, J., Buchloh, S., Wintermantel, E., Scharnweber, D. and Worch, H., "Non-destructive three-dimensional evaluation of biocompatible materials by microtomography using synchrotron radiation" Proc. SPIE 4503, 178--188 (2002)..

[15] Weitkamp, T., Diaz, A., David, C., Pfeiffer, F., Stampanoni, M., Cloetens, P. and Ziegler, E., "X-ray phase imaging with a grating interferometer", Optics Express 13 (16), 6296-6304 (2005).

[16] Reina-de la Torre, F., Rodriguez-Baeza, A. and Sahuquillo-Barris, J., "Morphological Characteristics and Distribution Pattern of the Arterial Vessels in Human Cerebral Cortex:A Scanning Electron Microscope Study", Anatomical Record 251 (1), 87-96 (1998).

[17] Dominietto, M., Friess, S., Grabherr, S., Herzen, J., Beckmann, F. and Müller, B., "Vessel Tree Visualization with Synchrotron Radiation-based Micro Computed Tomography", Annual Rep. HASYLAB at DESY 2007, 1559-1560 (2007).

[18] Bonse, U. and Busch, F., "X-ray computed microtomography ( $\mu \mathrm{CT})$ using synchrotron radiation (SR)", Prog. Biophys. molec. Biol. 65 (1/2), 133-169 (1996). 